\title{
Association of BrERF72 with methyl jasmonate-induced leaf senescence of Chinese flowering cabbage through activating JA biosynthesis-related genes
}

Xiao-li Tan ${ }^{1}$, Zhong-qi Fan', Wei Shan ${ }^{1}$, Xue-ren Yin², Jian-fei Kuang $\mathbb{B}^{1}$, Wang-jin Lu and Jian-ye Chen (1)

\begin{abstract}
The ethylene response factor (ERF) and phytohormone jasmonate (JA) are reported to function in leaf senescence. The involvement of ERF in JA-mediated leaf senescence, however, needs to be elucidated. In the present work, we demonstrate a Chinese flowering cabbage ERF transcription factor (TF), BrERF72, that is associated with JA-promoted leaf senescence. Exogenous application of methyl jasmonate (MeJA)-accelerated leaf senescence of Chinese flowering cabbage, evidenced by the data that MeJA treatment led to the stronger reduction in the maximum quantum yield (Fv/Fm), photosynthetic electron transport rate (ETR), and total chlorophyll content, while significant induction in the expression of several senescence-associated genes (SAGs) including BrSAG12, BrSAG19, and chlorophyll catabolic genes (CCGS) BrPAO1, BrNYC1, BrPPH1, and BrSGR1. Increases in levels of endogenous JA and transcripts of JA biosynthetic genes BrLOX4, BrAOC3, and BrOPR3 were also found after MeJA treatment. BrERF72 was a MeJA-inducible, nucleuslocalized protein, and possessed trans-activation ability. Transient overexpression of BrERF72 in tobacco leaves also promoted leaf senescence. More importantly, further experiments revealed that BrERF72 directly activated expression of BrLOX4, BrAOC3, and BrOPR3 through binding to their promoters via the GCC or DRE/CRT cis-element. Together, the novel JA-ERF association reported in our study uncovers a new insight into the transcriptional regulation of JA production mediated by ERF during JA-promoted leaf senescence in Chinese flowering cabbage.
\end{abstract}

\section{Introduction}

As the final phase of development, leaf senescence is considered as an important biological process in plants that nutrition substances in senescing leaves were reallocated to the newly formed organs, which can maximize plants viability in the next year ${ }^{1-3}$. However, precocious

\footnotetext{
Correspondence: J-y. Chen (chenjianye@scau.edu)

${ }^{1}$ State Key Laboratory for Conservation and Utilization of Subtropical Agrobioresources/Guangdong Provincial Key Laboratory of Postharvest Science of Fruits and Vegetables/Guangdong Vegetables Engineering Research Center, College of Horticulture, South China Agricultural University, 510642 Guangzhou, China

${ }^{2}$ The State Agriculture Ministry Laboratory of Horticultural Plant Growth, Development and Quality Improvement, College of Agriculture and Biotechnology, Zhejiang University, Zijingang Campus, 310058 Hangzhou, China
}

or abnormal senescence induced by stresses or other factors often shorten the growth stage of crops, resulting in a loss in crop yield and products quality ${ }^{4,5}$. In addition, leaf senescence limits the shelf-life and nutritional value of many leafy vegetables, representing the main postharvest problem of these vegetables ${ }^{6,7}$. Therefore, in agricultural aspects, understanding the regulatory mechanisms of leaf senescence is critical for the breeding of higher-yielding and well-nutrition crops, as well as for the better management to prevent postharvest loss.

The initiation and development of leaf senescence is tightly controlled by various endogenous and environmental factors, such as developmental stage, phytohormones, nutrients, and stresses ${ }^{2,8,9}$. Jasmonates (JAs), a group of oxylipin compounds ubiquitous in plants, act as 
a crucial signal to modulate diverse physiological processes including leaf senescence ${ }^{10}$. In Arabidopsis, the endogenous JA level increases dramatically during leaf senescence $^{11}$. Moreover, exogenously applied JA or its bioactive derivatives methyl jasmonate (MeJA) stimulate leaf senescence in several plants such as Arabidopsis ${ }^{11-14}$, wormwood $^{15}$, barley ${ }^{16}$, maize ${ }^{17}$, and rice ${ }^{18}$, by upregulating some senescence-associated genes (SAGs). Thus, JA is an important factor in inducing leaf senescence ${ }^{10,19}$.

During the past decades, great achievements have been made in JA biosynthesis, perception, and signaling in higher plants ${ }^{20-22}$. Briefly, JA and its derivatives are biosynthetized from $\alpha$-linolenic acid (18:3) ( $\alpha$-LeA), then $\alpha$ LeA is converted to 12-oxo-phytodienoic acid (12-OPDA) in several reactions catalyzed by 13-lipoxygenase (LOX), allene oxide synthase (AOS), and allene oxide cyclase (AOC), respectively. 12-OPDA is subsequently converted to JA by the involvement of OPDA reductase3 (OPR3) following three cycles of $B$-oxidation ${ }^{20,22}$. The JA signal is perceived by its receptor CORONATINE INSENSITIVE1 (COI1) to form a stable COI1/JA complex. This complex then interacts with JA ZIM-domain (JAZ) repressors to degrade JAZ through the $26 \mathrm{~S}$ proteasome pathway, and to liberate various downstream transcription factors $(\mathrm{TFs})^{20-22}$. These TFs, including $\mathrm{bHLH}^{14,23,24}, \mathrm{MYB}^{25}$, WD-bHLH-MYB transcription complex ${ }^{26}$, and WRKY ${ }^{13}$, are essential for JA-mediated physiological processes. Interestingly, a TEOSINTE BRANCHED/CYCLOIDEA/ PCF (TCP) TF TCP4 directly activates the expression of JA biosynthetic gene $L O X 2$, while the repression of TCP4 by miR319 suppresses JA biosynthesis and delays leaf senescence in Arabidopsis ${ }^{27}$. Another TCP TF TCP20 inhibits $L O X 2$ and the $t c p 20$ mutant exhibits an earlier onset of leaf senescence ${ }^{28}$. These data demonstrate that activation of JA biosynthesis induces senescence-related genes expression and promotes leaf senescence. Although JA biosynthesis and signaling networks have been intensively investigated, as well as leaf senescence promoted by JA, the upstream TFs involved in leaf senescence by controlling its level remain to be explored.

Despite large TF families such as NO APICAL MERISTEM/ARABIDOPSIS TRANSLATION ACTIVATIONFACTOR/CUP-SHAPED COTYLEDON (NAC), WRKY, bHLH, APETALA2/ETHYLENE RESPONSE FACTOR (AP2/ERF), and MYB are involved in leaf senescence, consisting of a hierarchical and coordinated regulatory network ${ }^{22,29,30}$, only several TFs, including WRKY53 $3^{31}$, WRKY54 and WRKY70 ${ }^{32}$, WRKY $57^{13}$, ANAC092/ANAC019/055/072 ${ }^{19}$, bHLH03/bHLH13/ bHLH14/bHLH17 $7^{14}$, and MYC2/MYC3/MYC4 ${ }^{19}$, are identified to be positively or negatively involved in JApromoted leaf senescence. Many studies have demonstrated that AP2/ERF TFs not only act as important regulators of ethylene signaling ${ }^{33}$, but also are involved in many physiological processes controlled by $\mathrm{JA}^{34}$. Yet, little is known about the regulatory mechanisms of AP2/ERFs associated with JA-promoted leaf senescence.

Leafy vegetables like Chinese flowering cabbage/choy sum are rich in nutraceutical and health-promoting metabolites $^{35}$. But leafy vegetables senesce rapidly after harvest, resulting in substantial loss and short shelf-life due to yellow leaves. Our previous study showed that a leaf senescence-induced TF BrWRKY65 directly activated three $S A G s$ in Chinese flowering cabbage ${ }^{7}$. Nevertheless, the underlying transcriptional regulation of Chinese flowering cabbage leaf senescence is still largely unclear. Here, we reported that MeJA pretreatment accelerated leaf senescence of Chinese flowering cabbage. More importantly, we further identified a novel transcriptional activator AP2/ERF TF BrERF72 that associated with leaf senescence by directly regulating JA biosynthetic genes $B r L O X 4, B r A O C 3$, and BrOPR3. Our findings thus provide novel evidence about the transcriptional regulation of JA-mediated leaf senescence involving ERF TFs.

\section{Materials and methods}

\section{Plant materials and treatments}

Chinese flowering cabbages (Brassica rapa var. parachinensis) were harvested after $\sim 40$ days of growth. These harvested cabbages were pre-cooled and transported to the lab immediately. Uniform and no damaged cabbages were chosen and randomly divided into two groups for the following treatments.

For MeJA treatment, the cabbages were sprayed with $100 \mu \mathrm{M}$ MeJA (containing 0.2\% Tween-20). The cabbages sprayed with distilled water were served as control. Both control and MeJA-treated cabbages were subsequently put into plastic baskets (20 per box), which were packed with polyethylene-perforated plastic bags $(0.03 \mathrm{~mm}$ thickness, with six holes of $1 \mathrm{~cm}$ in diameter at each side), and stored in the incubators at $15^{\circ} \mathrm{C}$. Immediately after MeJA treatment and after 1, 3, 5, and 7 days of storage, the second leaf from the bottom of ten cabbages were collected for physiological and molecular analysis. The collected leaves were rinsed three times with distilled water containing $0.2 \%$ Tween-20 to remove the residual MeJA on the leaf surface, frozen in liquid nitrogen, and stored at $-80^{\circ} \mathrm{C}$ for further use.

\section{Leaf senescence evaluation}

Senescence-associated physiological markers, such as total chlorophyll content and chlorophyll fluorescence, such as the maximal PS II quantum yield $(\mathrm{Fv} / \mathrm{Fm})$ and photosynthetic electron transport rate (ETR), were measured to evaluate leaf senescence. Leaf tissues $(\sim 2.5 \mathrm{~g})$ were ground to power using liquid nitrogen, and total chlorophyll was extracted with $80 \%$ cold acetone overnight at $4{ }^{\circ} \mathrm{C}$, and then estimated spectrophotometrically 
at 663 and $645 \mathrm{~nm}^{36} . \mathrm{Fv} / \mathrm{Fm}$ and ETR were calculated non-invasively using an Imaging-PAM-M series chlorophyll fluorometer (Heinz Walz, Effeltrich, Germany) integrated with a CCD camera that enables to capture digital images of the emitted fluorescence with high resolution ${ }^{37}$.

\section{Quantification of endogenous JA content by enzyme- linked immunosorbent assay (ELISA)}

About $1.0 \mathrm{~g}$ of cabbage leaf was used for the analysis. The extraction of JA and measurement of ELISA using monoclonal antibodies of JA were performed as described in previous studies ${ }^{38}$.

\section{RNA extraction, CDNA synthesis, gene cloning, and sequence analysis}

Total RNA of sampled leaves was isolated using the RNeasy Plant Mini Kit (Qiagen, Germany). cDNA used as the template for reverse transcription polymerase chain reaction (RT-PCR) and quantitative real-time PCR (qRTPCR) was synthesized from $1 \mu \mathrm{g}$ of total RNA using a PrimeScript ${ }^{\text {TM }}$ RT reagent kit (Takara, Japan). According to Chinese cabbage chiifu genome in BRAD (http:// brassicadb.org/brad/), the coding region of BrERF72 was cloned through RT-PCR with gene-specific primers. PCR products were sequenced and blasted in the NCBI database for the homology identification. Basic information of BrERF72 protein such as theoretical isoelectric point (pI) and mass value was calculated using the online database (http://web.expasy.org/compute_pi/). CLUSTALW (version 1.83), GeneDoc, and MEGA5 software were used for the alignment and phylogenetic tree of AP2/ERF proteins, respectively.

\section{Analysis of gene expression with qRT-PCR}

qRT-PCR was carried out using GoTaq ${ }^{\circledR}$ qPCR Master Mix Kit (Promega, USA) in a Bio-Rad CFX96 Real-Time PCR System (Bio-Rad, USA). EF-1- $\alpha$ (GO479260) was adopted as the reference gene to normalize the transcript levels of each gene, using the cycle threshold $(C t)$ value.

\section{Subcellular localization analysis}

The full length of BrERF72 without stop codon was subcloned into the pEAQ-GFP vector ${ }^{39}$ to fuse with the green fluorescent protein (GFP) gene sequence. Then, the pEAQ-BrERF72-GFP or the control pEAQ-GFP vector was transferred into the Agrobacterium tumefaciens strain GV3101 by electroporation, and transiently expressed in tobacco (Nicotiana benthamiana) leaves as described previously ${ }^{40}$. After $48 \mathrm{~h}$ of infiltration, infected leaf tissues were collected for analysis. GFP signal was captured using a fluorescence microscope (Zeiss Axioskop 2 Plus).

\section{Transcriptional activation analysis in yeast cells}

First, BrERF72 was inserted into the pGBKT7 vector (Clontech, USA) to fuse with GAL4 DNA-binding domain. Then, the fusion construct pGBKT7-BrERF72, positive control (pGBKT7-53 + pGADT7-T), and negative control (pGBKT7 vector) were transformed into yeast cells Gold $\mathrm{Y} 2 \mathrm{H}$ using the lithium acetate method. The transcriptional activation ability of BrERF72 was evaluated based on the growth status and $\alpha$-galactosidase activity of yeast cells that grow on SD medium without tryptophan (SD/-Trp), or tryptophan, histidine, and adenine (SD/Trp-His-Ade).

\section{Promoter analysis}

DNeasy Plant Mini Kit (Qiagen, Germany) was used to isolate genomic DNA of cabbage leaves. The promoters of three JA biosynthetic genes BrLOX4, BrAOC3, and BrOPR3 were amplified with nested PCR using a Genome Walker Kit (Clontech). Conserved cis-element motifs presented in each promoter was predicted in Plant-CARE database (http://bioinformatics.psb.ugent.be/webtools/ plantcare/html/).

\section{Electrophoretic mobility shift assay (EMSA)}

The coding sequence of BrERF72 was subcloned into the pGEX-4T-1 (Amersham Biosciences) vector to produce recombinant GST-BrERF72 protein in Escherichia coli strain BM Rosetta (DE3). The induced GST-BrERF72 protein was further purified using glutathione-superflow resin (Clontech) according to the manufacturer's protocol. The $5^{\prime}$ ends of synthesized oligonucleotide probes were labeled with biotin. EMSA was performed using the LightShift Chemiluminescent EMSA Kit (Thermo Scientific) as previously described ${ }^{7,40}$. Briefly, GST-BrERF72 protein and biotin-labeled probes were incubated together, then free and protein-DNA complexes were separated by $6 \%$ native polyacrylamide gel electrophoresis, transferred onto nylon membrane and detected by a ChemiDoc $^{\text {Tw }}$ MP Imaging System (Bio-Rad, USA) using the chemiluminescence method. Unlabeled and mutated probes, as well as GST protein alone, were used as competitors and negative control, respectively.

\section{Dual-luciferase reporter assays in tobacco leaves}

For transcriptional activity analysis of BrERF72, its coding sequence was cloned into the $35 \mathrm{~S}$ promoter-drove $\mathrm{pBD}$ vector to fuse with the yeast GAL4 DNA-binding domain (GAL4BD) as effector (pBD-BrERF72). The double-reporter vector contains a firefly luciferase (LUC) driven by five copies of the GAL4-binding element $(5 \times$ GAL4) and minimal TATA region of CaMV 35S. Renilla luciferase (REN) in the same vector drove by the $35 \mathrm{~S}$ promoter was used for normalization. For assaying the binding activity of BrERF72 to the promoters of BrLOX4, 
$B r A O C 3$, and $B r O P R 3$, their promoters were inserted into pGreenII 0800-LUC double-reporter plasmid as reporters ${ }^{41}$. Effector plasmid was constructed by inserting BrERF72 into the pEAQ vector. The constructed effectors and reporters plasmids with different combinations were co-transformed into tobacco leaves mentioned above.

Activities of LUC and REN luciferase were quantified after $48 \mathrm{~h}$ of infiltration using the dual luciferase assay kit (Promega, USA) on the Luminoskan Ascent Microplate Luminometer (Thermo Fisher Scientific, USA). The transcriptional ability of BrERF72 was assessed by the LUC to REN ratio. Six biological repeats were included for each pair.

\section{Transient overexpression of BrERF72 in tobacco leaves}

Transient overexpression of pEAQ-BrERF72 mentioned above were performed in tobacco (Nicotiana tabacum) leaves as described previously ${ }^{42,43}$. After infiltration, tobacco plants were maintained in the chamber and the infiltrated leaves were sampled for photos.

\section{Data analysis}

Data were recorded as mean \pm standard errors (SE) of three or six independent biological replicates. Statistical differences between samples $(P<0.05$ or 0.01$)$ were determined by Student's $t$ test.

\section{Primers}

All primers designed and used in this work are presented in Supplementary Table S1.

\section{Results}

Exogenous application of MeJA promotes leaf senescence of Chinese flowering cabbage

The leaves of control and MeJA-treated cabbages started to turn yellow after 5 and 3 days of storage, respectively, and became serious thereafter. Furthermore, the leaves of MeJA-treated cabbages showed more obvious yellowing than control leaves during senescence (Fig. 1a). Harvested green leaves still have the ability of photosynthesis, which is affected by senescence. To monitor spatial and temporal changes in photosynthetic parameters in leaves, noninvasive chlorophyll fluorescence imaging was used. In agreement with more yellow areas developed in MeJA-treated leaves, the maximum quantum yield $(\mathrm{Fv} / \mathrm{Fm})$ and photosynthetic electron transport rate (ETR) reduced faster and stronger in the MeJAtreated leaves than those of the control (Fig. 1a, b). Accordingly, total chlorophyll content was obviously lower in MeJA-treated leaves (Fig. 1b). We also compared the expression of SAGs including BrSAG12, BrSAG19, and chlorophyll catabolic genes (CCGs) such as BrPAO1, $B r N Y C 1, B r P P H 1$, and BrSGR1 encoding pheophorbide a oxygenase (PAO), NON-YELLOW COLORING (NYC), pheophytin pheophorbide hydrolase (PPH), and STAYGREEN (SGR), respectively, between MeJA-treated and control leaves. As shown in Fig. 1c, transcript levels of all $S A G s$ were obviously increased during leaf senescence. Moreover, MeJA treatment obviously increased the transcripts of those genes compared to control, with a 1.18-, 1.67-, 1.29-, 1.48-, and 1.41-fold induction for BrSAG12, $B r S A G 19, B r N Y C 1, B r P P H 1$, and BrSGR1 at 7 days, and a 1.21-fold induction for BrPAO1 at 5 days of storage, respectively (Fig. 1c). Therefore, all of the results above imply that exogenous application of MeJA promotes leaf senescence of Chinese flowering cabbage.

\section{Exogenous MeJA increases endogenous level of JA and enhances expression of JA biosynthetic genes BrLOX4, $B r A O C 3$, and BrOPR3}

To test whether endogenous JA level was changed by exogenous application of MeJA, we measured the JA concentration in cabbage leaves during senescence. The endogenous JA in control or MeJA-treated leaves accumulated during senescence, and MeJA-treated leaves had significantly higher level than the control (Fig. 2a). At 5 days of storage, the JA level in leaves of MeJA treatment was $561.19 \mathrm{ng} / \mathrm{mg} \mathrm{FW}$, which was about 0.86 -fold higher than that of the control leaves (301.24 ng/mg FW) (Fig. 2a). We further speculated that JA biosynthetic gene expressions were influenced by MeJA treatment. As expected, qRT-PCR analysis showed that consistent with the change of JA content, transcription levels of JA biosynthetic genes, including BrLOX4, BrAOC3, and $B r O P R 3$, were significantly upregulated in MeJA-treated leaves (Fig. 2b). Thus, it appears that exogenous MeJA increases endogenous concentration of JA during leaf senescence, possibly through induction of JA biosynthetic genes.

\section{Identification and molecular characterization of BrERF72}

According to our RNA-seq and Chinese cabbage genome (http://brassicadb.org/brad/) databases, notably, a putative AP2/ERF gene (XM_009137250.2) was notified to be obviously induced by leaf senescence. Homology search revealed that its sequence shared the highest identity with Arabidopsis thaliana AtERF72 (79\%), thus it was termed as BrERF72. The length of open reading frame (ORF) of BrERF72 is $738 \mathrm{bp}$, which encodes a protein of 245 amino acids. Its predicted molecular weight and isoelectric point (pI) of BrERF72 are $27.42 \mathrm{kDa}$ and 4.94, respectively. Sequence alignment showed that BrERF72 contains one highly conserved AP2 DNA-binding domain, containing $\sim 60$ amino acids from 80 to 137 aa (Fig. 3a). The conserved 14th adenine (A) and 19th aspartate (D), which are vital for DNA-binding sequences recognition $^{44}$, were also found in BrERF72 (Fig. 3a). To know the classification of BrERF72, a phylogenetic tree 

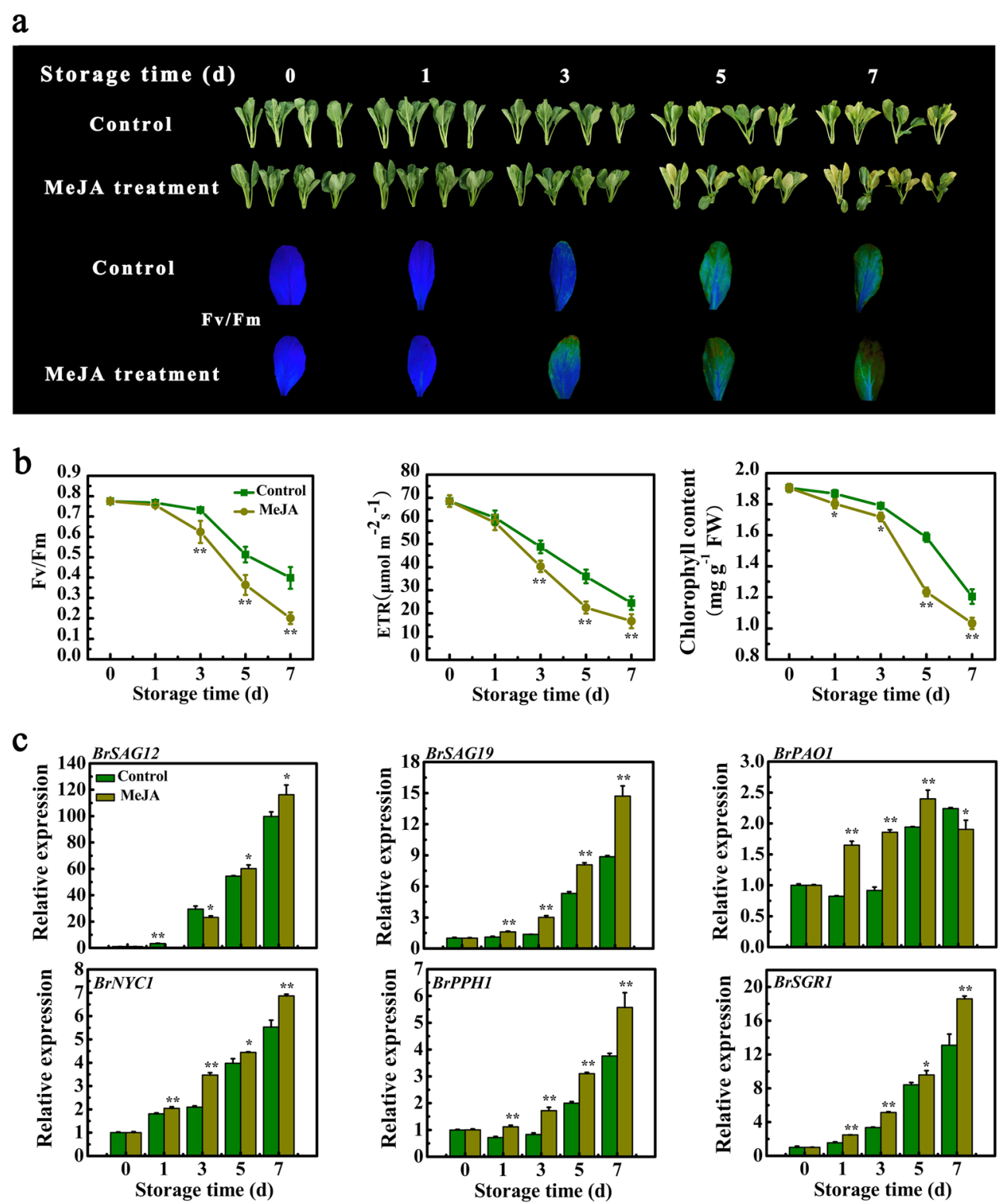

Fig. 1 Application of MeJA promotes leaf senescence of Chinese flowering cabbage. a Appearance and chlorophyll fluorescence imaging with Fv/Fm of control and MeJA-treated cabbage leaves during senescence. b Changes of Fv/Fm, ETR, and chlorophyll content in control and MeJAtreated cabbage leaves during senescence. c Relative expression of six SAGs including BrSAG12, BrSAG19, BrPAO1, BrNYC1, BrPPH1, and BrSGR1 in control and MeJA-treated cabbage leaves during senescence. Data presented in $\mathbf{b}$ and $\mathbf{c}$ are the mean $\pm \mathrm{SE}$ of three biological replicates. Asterisks indicate a significant difference in MeJA-treated leaves compared with control leaves (Student's $t$ test: ${ }^{*} P<0.05$ and ${ }^{* *} P<0.01$ )

was constructed, showing that BrERF72 is closely related to AtERF72, and both are members of ERF subfamily group B-2 (Fig. 3b).

Similar with the RNA-seq data, qRT-PCR analysis showed that expression of BrERF72 was induced during leaf senescence. Moreover, more remarkably increase of BrERF72 transcript was observed in MeJA-treated leaves, with $\sim 0.9$ - and 0.5 -fold higher than that of the control leaves at 5 and 7 days of treatment, respectively (Fig. 4a). To know the subcellular localization of BrERF72, a
BrERF72 and green fluorescent protein (GFP) fusion construct was prepared, and transiently expressed in tobacco leaves. The fluorescence of BrERF72-GFP was predominately observed in the nucleus of epidermal cells, but GFP signal of positive control was found both in cytosol and nucleus (Fig. 4b). To examine the transcriptional activity of BrERF72, a BrERF72 fusing with the GAL4 DNA-binding domain-coding sequence (GAL4BD) construct was prepared, and introduced into yeast cells. Like the positive control (p53 $+\mathrm{T}$-antigen), yeast cells 
a

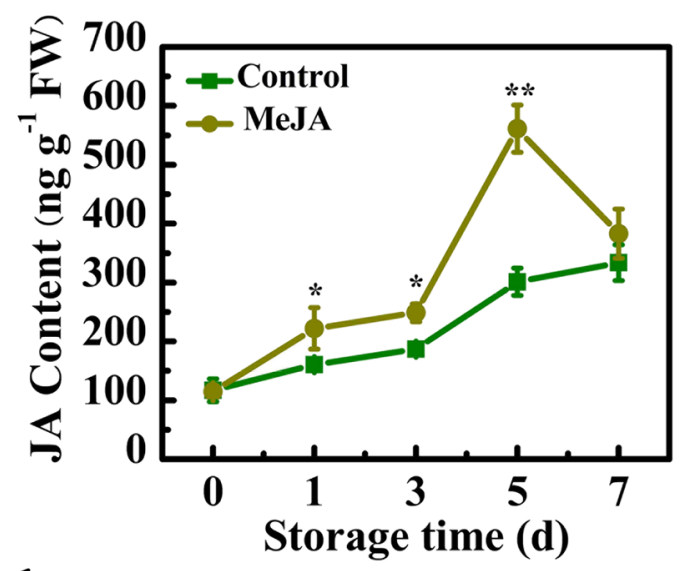

b
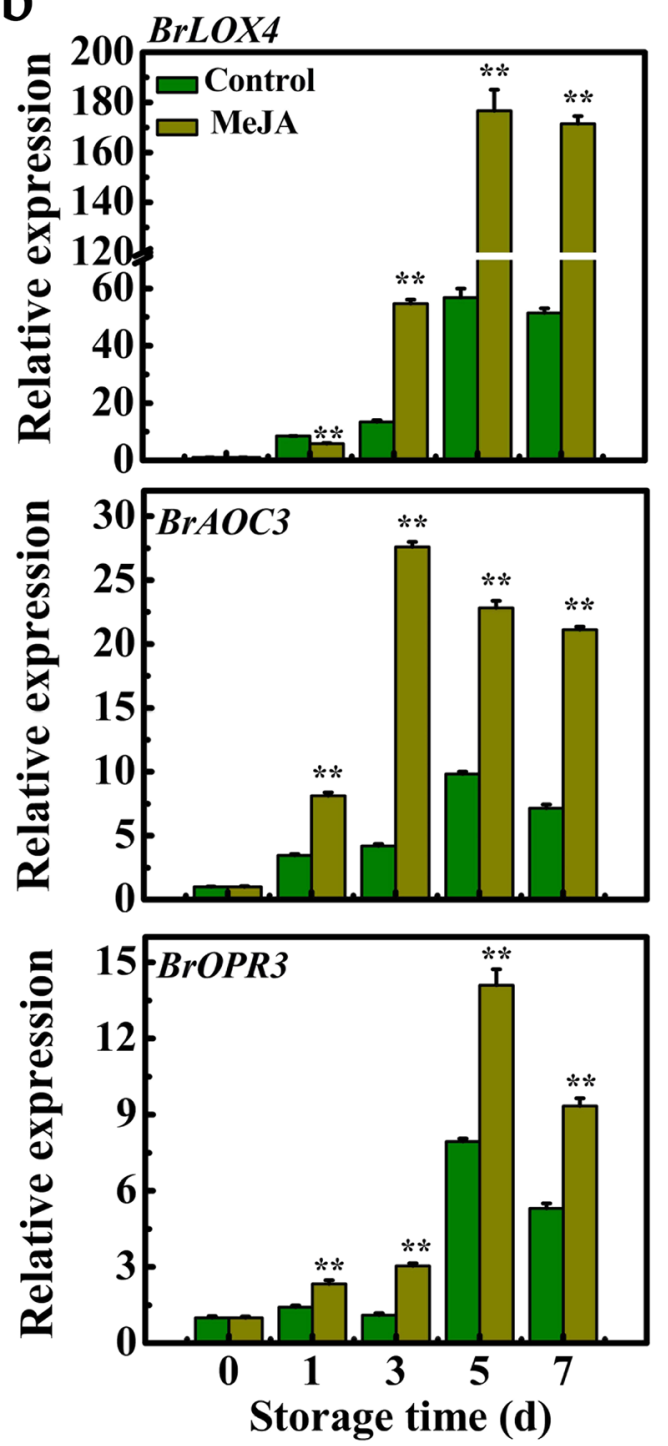

Fig. 2 Application of MeJA increases endogenous JA level and enhances expression of JA biosynthetic genes during leaf senescence of Chinese flowering cabbage. a Changes of endogenous JA level. $\mathbf{b}$ Expression of JA biosynthetic genes including BrLOX4, BrAOC3, and BrOPR3. Each value represents the mean \pm S.E. of three biological replicates. Asterisks indicate a significant difference between control and MeJA treatment by student's $t$ test: ${ }^{*} P<0.05$ and ${ }^{*} P<0.01$

expressing the BrERF72 grew well on SD plates without tryptophan, histidine, and adenine, and showed $\alpha$ galactosidase activity (Fig. 4c), indicating that BrERF72 possesses trans-activation ability in yeast cells. Using the dual-luciferase reporter system, trans-activation of BrERF72 was further verified in tobacco leaves, which showed that expression of BrERF72 with the dualluciferase reporter, as well as the transcriptional activator control VP16, remarkably elevated the LUC/REN ratio (Fig. 4d). Collectively, these data demonstrate that BrERF72 is a MeJA-induced transcriptional activator.

\section{Transient overexpression of BrERF72 in tobacco leaves} leads to accelerated leaf senescence

The possible association of BrERF72 with leaf senescence was further verified through the transient overexpression in tobacco leaves. As shown in Fig. 5, transient overexpression of BrERF72 led to acceleration of leaf senescence, since more yellow lesions (the right blade of the leaves) were appeared, compared with the empty vector (the left blade of the leaves) (Fig. 5).

\section{BrERF72 directly targets $B r L O X 4, B r A O C 3$, and BrOPR3 promoters through GCC or DRE/CRT elements}

Based on these above results, the regulatory mechanism of BrERF72 associated with MeJA-induced leaf senescence, especially whether BrERF72 can directly target JA biosynthetic genes, attracts our attention. Previously, it was shown that ERF TFs specifically bind directly to both GCC-box (AGCCGCC) and DRE/CRT [(A/G)CCGAC] motifs $^{18,45,46}$. EMSA using the purified recombinant BrERF72 protein (Fig. 6a) was performed to investigate whether BrERF72 could recognize GCC-box and DRE/ CRT motifs. As shown in Fig. 6b, the recombinant BrERF72 protein could directly bind to GCC-box and DRE/CRT motifs. Intriguingly, DRE/CRT motif was found in BrLOX4 and BrAOC3 promoters, and GCC-box motif was observed in BrOPR3 promoters (Supplementary Text S1). Consistently, it was shown that the recombinant BrERF72 protein could bind directly to the DRE/CRT motif-containing fragment of the $\mathrm{BrLOX} 4$ and $\mathrm{BrAOC} 3$ promoters, and the GCC-box-containing fragment of the BrOPR3 promoter, respectively (Fig. 6c). Moreover, increasing amounts of unlabeled wild probe obviously reduced BrERF72 binding to the biotin-labeled probes, 


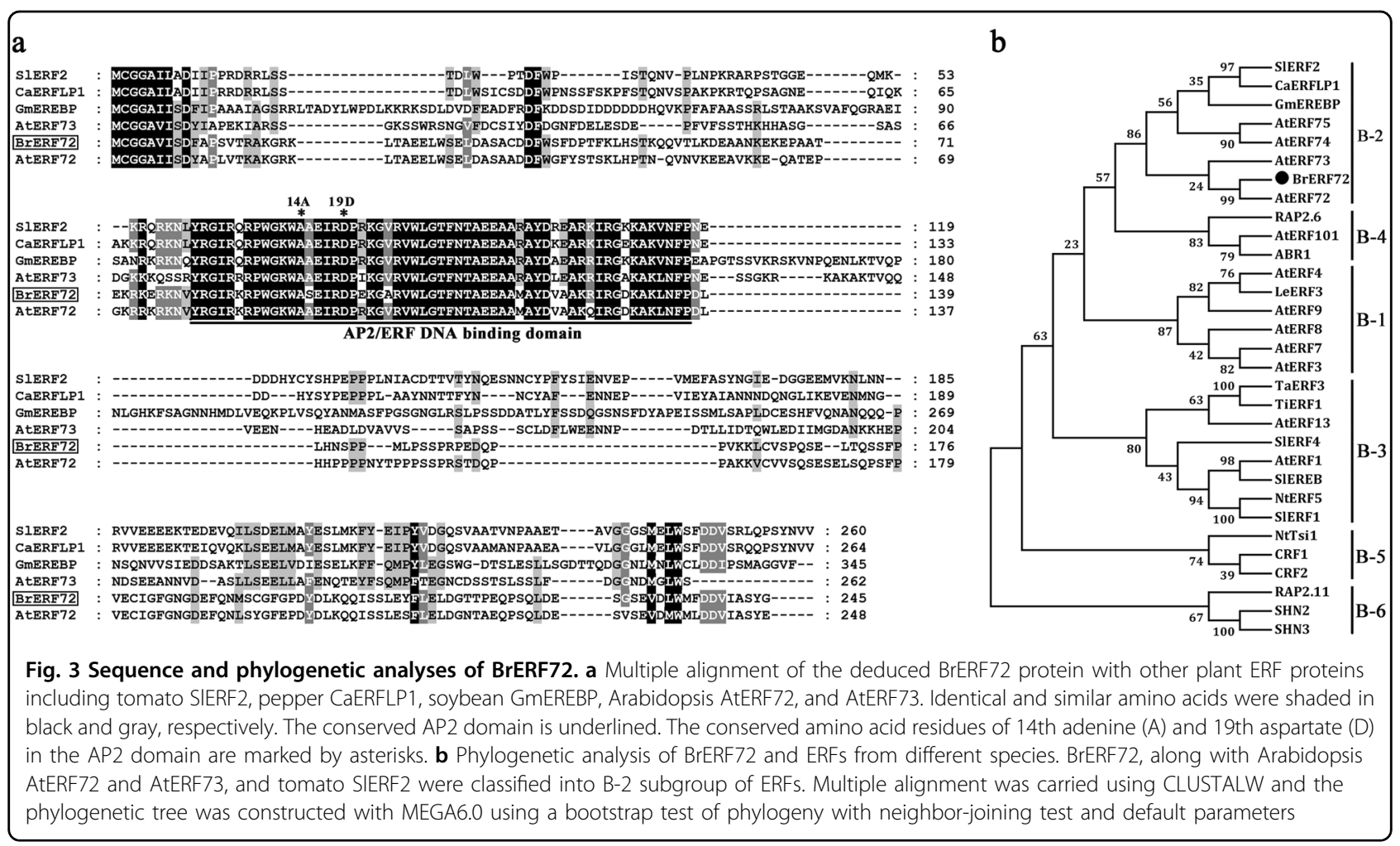

whereas unlabeled mutant probes were failed to compete for BrERF72 binding (Fig. 6c), further indicating that BrERF72 directly targets the BrLOX4, BrAOC3, or $B r O P R 3$ promoters via the DRE/CRT or GCC-box motifs.

\section{BrERF72 activates the expression of BrLOX4, BrAOC3, and BrOPR3}

To detect the effect of BrERF72 on the expression of $B r L O X 4, B r A O C 3$, and BrOPR3 after binding to their promoters, we performed dual-luciferase assays in tobacco leaves. $\mathrm{BrLOX} 4, \mathrm{BrAOC} 3$, or $\mathrm{BrOPR} 3$ promoter was inserted into the pGreenII 0800-LUC plasmid and coinfiltrated into tobacco leaves with BrERF72 (Fig. 7a). As illustrated in Fig. 6b, when BrERF72 was co-transformed with $B r L O X 4, B r A O C 3$, or BrOPR3 pro-LUC reporter, the value of LUC/REN ratio was remarkably increased, compared with the empty control. These data clearly indicate that BrERF72 activates BrLOX4, BrAOC3, and $B r O P R 3$ expression by directly targeting their promoters.

\section{Discussion}

The phytohormone JA has been reported to promote leaf senescence in several species ${ }^{10,19}$. Our study also showed that exogenous MeJA treatment promoted leaf senescence of Chinese flowering cabbage, as MeJA treatment caused more reduction in $\mathrm{Fv} / \mathrm{Fm}$, ETR, and total chlorophyll content, while enhancing the expression of BrSAG19, and BrPAO1, BrNYC1, BrPPH1, and BrSGR1, resulting in more serious yellowing in MeJA-treated cabbage leaves (Fig. 1).

Several large TF families, including WRKY, NAC, and bHLH, are implicated in regulating JA-induced leaf senescence ${ }^{13,14,19,47}$. As one of the largest plant-specific TF families, AP2/ERF TFs differentially regulate diverse aspects of JA-mediated physiological processes ${ }^{22,34}$, for example, Catharanthus roseus ORCA3 is a MeJAresponsive AP2/ERF TF, and involved in JAs-stimulating monoterpenoid indole alkaloid biosynthesis ${ }^{48}$. Two AP2/ ERF TFs AaERF1 and AaERF2 of Artemisia annua act as positive regulators of JAs-induced artemisinin biosynthesis $^{49}$. AP2/ERF TF members also positively or negatively initiate the onset of leaf senescence in Arabidopsis, rice and tomato ${ }^{29}$. While their involvement in JA-promoted leaf senescence has been unclear. In this study, we identified a MeJA-inducible B-2 subgroup of ERF TF BrERF72 in Chinese flowering cabbage, and provided evidence showing that BrERF72 may be associated with MeJApromoting leaf senescence through the regulation of transcription of JA biosynthetic genes $B r L O X 4, B r A O C 3$, and $\mathrm{BrOPR} 3$.

Accumulating evidence has supported the necessity of intact JA signaling for JA-accelerated leaf senescence $^{10,11,13,14,19,50,51}$. JAZ (JASMONATE ZIMDOMAIN) proteins and bHLH TFs MYCs are two key components of JA signaling, and their pivotal roles in JApromoted leaf senescence have been extensively 


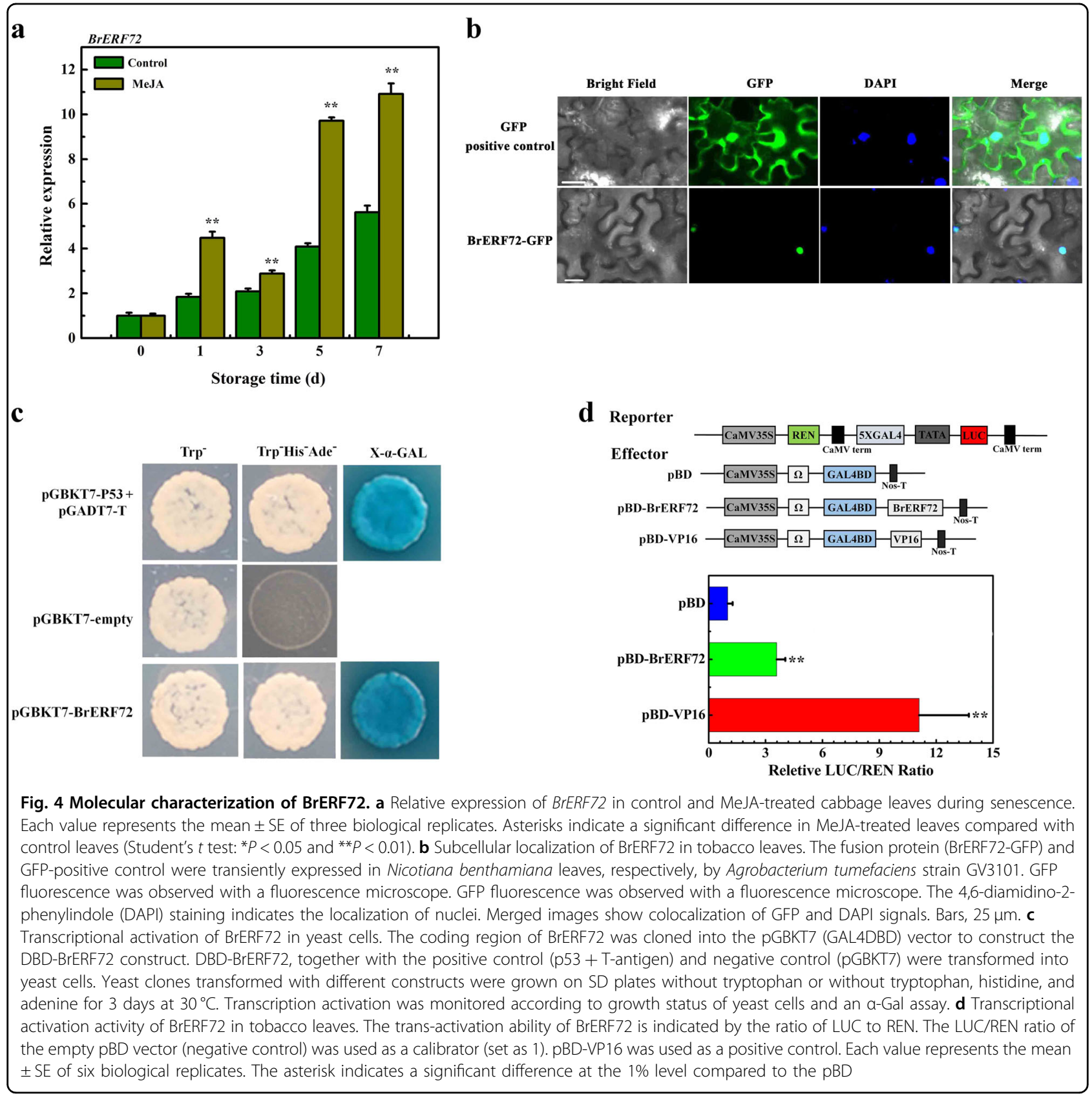

studied $^{10,50}$. During senescence, endogenous JA content and expression of JA biosynthetic genes are strongly enhanced in senescent leaves and by MeJA treatment ${ }^{11}$. Similar results were also obtained in our study (Fig. 2). Interestingly, Arabidopsis class II CINCINNATA (CIN)/ TCP TFs such as TCP4 positively influence JA biosynthesis through directly activating the transcription of $L O X 2^{27}$. miR319a/JAGGED and WAVY (jaw) can target class II TCPs ${ }^{52}$, and consequently LOX2 expression and JA level in leaves of jaw-D plants are low, which leads to attenuate JA-induced senescence, while exogenous MeJA application rescues the senescence phenotype of jaw-D plants $^{27}$. Recently, the class I TCP members TCP9 and TCP20 are shown to repress LOX2 expression, and the tcp $9 t c p 20$ double mutants exhibit an earlier initiation of leaf senescence ${ }^{28}$. In this work, we found that the Chinese flowering cabbage AP2/ERF TF BrERF72 was induced by MeJA, localized in the nucleus and possessed transcriptional activation ability (Fig. 4). Transient overexpression of BrERF72 in tobacco leaves also resulted in accelerated leaf senescence (Fig. 5). More importantly, BrERF72 activated the transcription of three JA biosynthetic genes $B r L O X 4, B r A O C 3$, and BrOPR3 by directly targeting their promoters (Figs. 6 and 7). Our results, together with 


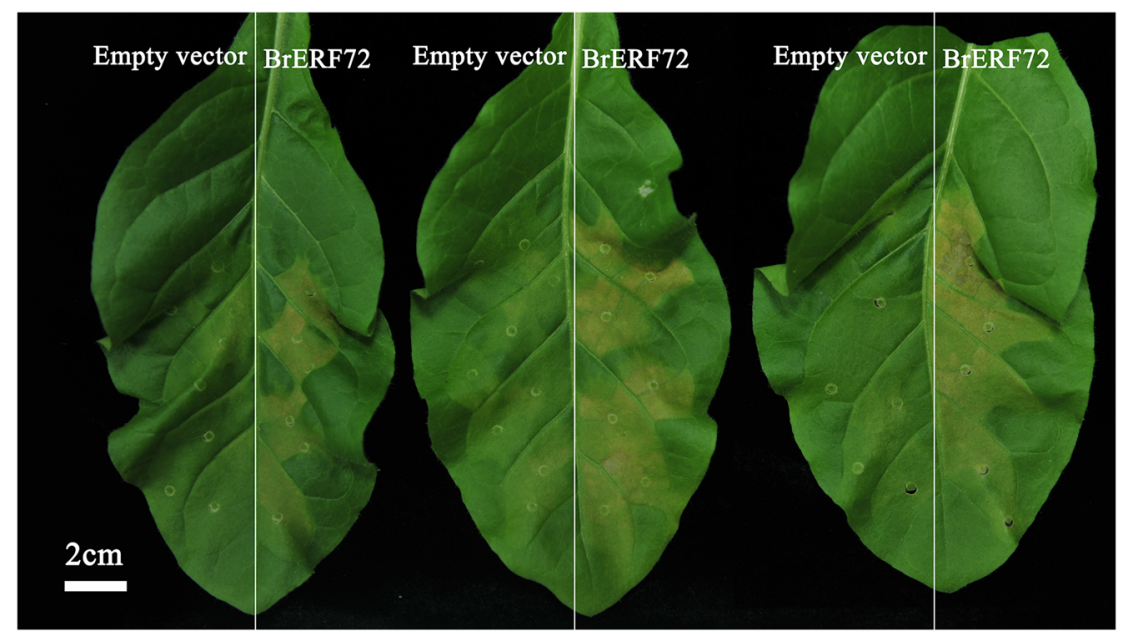

Fig. 5 Transient overexpression of BrERF72 promotes leaf senescence in Nicotiana tabacum. Appearance of Nicotiana tabacum leaves transiently expressed with empty vector (left side of leaf) and BrERF72 (right). Three independent leaves were imaged at 5 days after infiltration

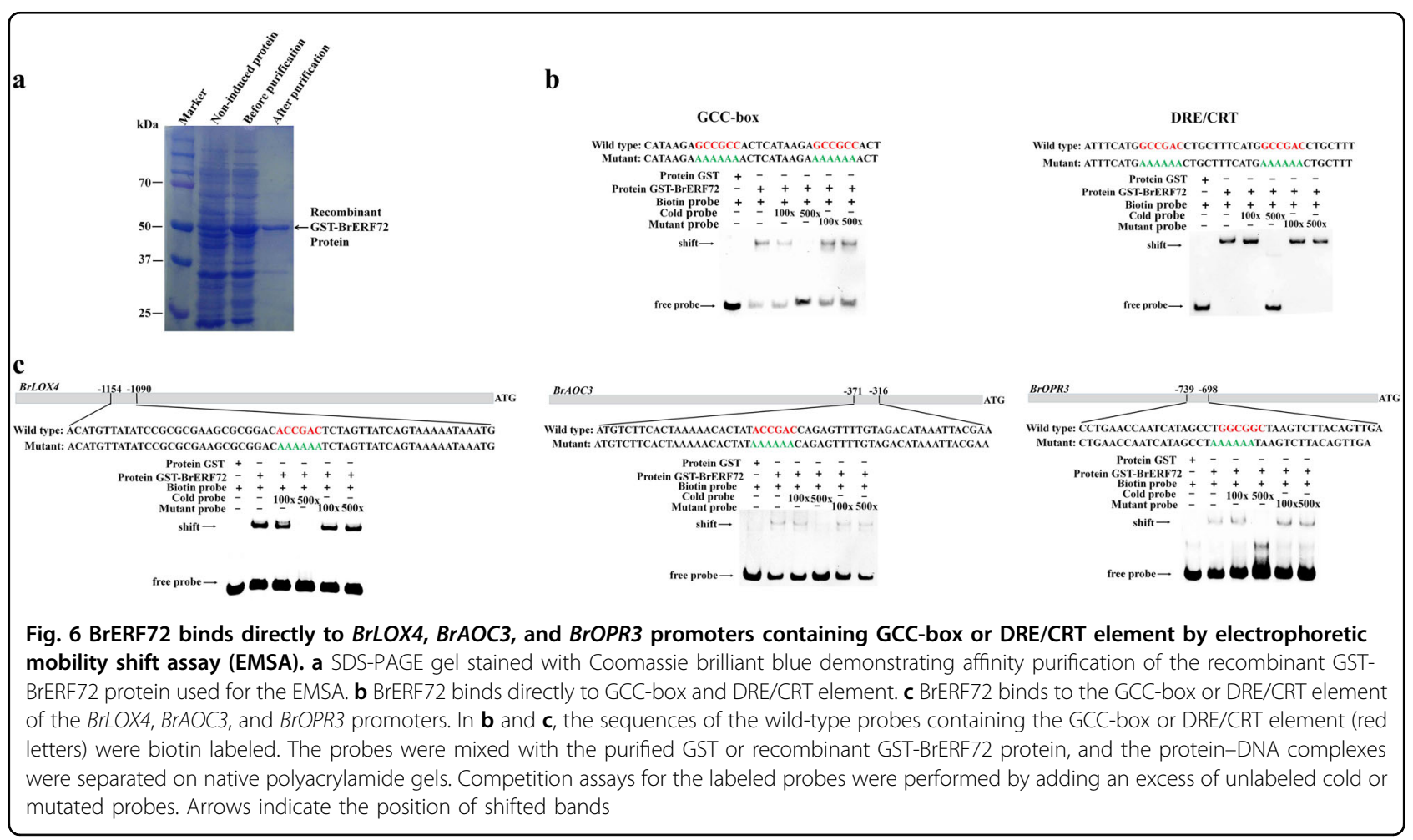

previous reports, reveal that besides intact JA signaling, transcriptional regulation of JA biosynthesis is also important for JA-modulated leaf senescence. However, it should be pointed out that transgenetic research such as stable overexpression of BrERF72 in Arabidopsis will be helpful to fully elucidate the involvement of BrERF72 in JA-promoted leaf senescence of Chinese flowering cabbage. Intriguingly, recently it is reported that a JA-induced TF MdMYC2 functions in JA-promoting ethylene biosynthesis via directly targeting MdERFs and ethylene biosynthetic genes MdACS1 and MdACO1 during apple fruit ripening ${ }^{53}$. Together with our work, these findings demonstrate that TFs, such as ERF and MYC2, are involved in crosstalk between JA and ethylene in mediating biological process.

Leaf yellowing, causing by chlorophyll degradation controlled by CCGs, is the most distinguishing feature of senescence. Recently, it was showed that the citrus 


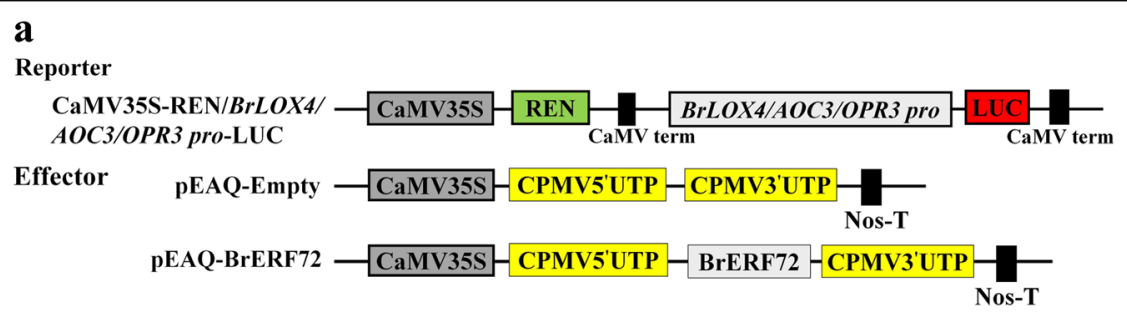

b

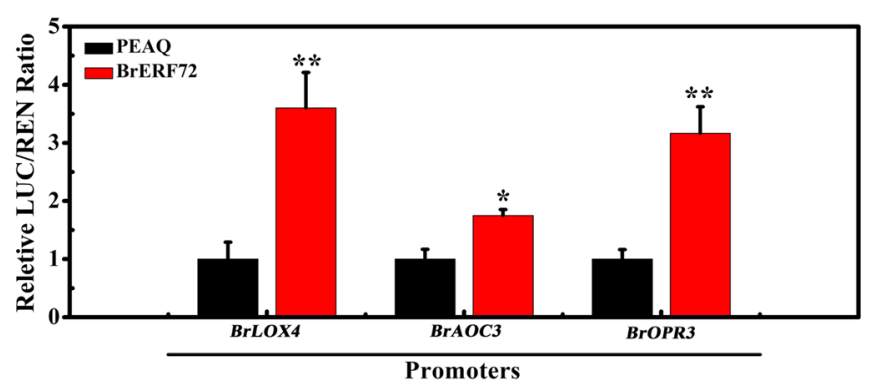

Fig. 7 Dual-luciferase transient expression assay in tobacco leaves showing that BrERF72 activates the expression of $B r L O X 4, B r A O C 3$, and BrOPR3. a Diagrams of the reporter and effector vectors. b BrERF72 activates BrLOX4, BrAOC3, and BrOPR3 promoters. Each value represents the mean \pm SE of six biological replicates. Asterisks indicate significantly different values (Student's $t$ test: ${ }^{*} P<0.05$ and ${ }^{* *} P<0.01$ )

CitERF13 directly targets CitPPH promoter and activates its expression, thereby leading to the acceleration of chlorophyll degradation and fruit degreening ${ }^{42}$. Three bHLH TFs of Arabidopsis MYC2/3/4, and three NAC TFs ANAC019/055/072, are involved in JA-promoted degreening via the direct mediation of a set of CCGs (NYC1 and $S G R s)^{19}$. Furthermore, MYC2 interacts with ANAC019 and they act synergistically to activate SGR1 expression ${ }^{19}$. Leaf senescence is believed to be a complicated biological process concerning of many transcriptional regulators ${ }^{54}$. Therefore, identifying other targets such as CCGs of BrERF72, and dissecting other JA-responsive TFs such as MYCs, NACs, and WRKYs, as well as their connections in association with JA-mediated leaf senescence of Chinese flowering cabbage, will be our intriguing research topics in the future, which might extend our knowledge of the transcriptional regulation of leaf senescence in Chinese flowering cabbage.

\section{Conclusions}

Taken together, we demonstrated that application of MeJA promoted leaf senescence of Chinese flowering cabbage. Furthermore, we characterized a MeJAinducible transcriptional activator BrERF72, and showed that BrERF72 activated three JA biosynthetic gene expressions via directly targeting their promoters. We propose that BrERF72 might be associated with MeJA-prompted leaf senescence of Chinese flowering cabbage through directly regulating JA biosynthetic genes. Our findings provide novel cues about the transcriptional regulation of JA biosynthesis involving ERF TFs associated with JA-mediated leaf senescence.

\section{Acknowledgements}

We are grateful to Dr. George P. Lomonossoff (Department of Biological Chemistry, John Innes Centre, Norwich Research Park) for providing the pEAQ vectors. This study was funded by National Natural Science Foundation of China (Grant no. 31671897).

\section{Author details}

${ }^{1}$ State Key Laboratory for Conservation and Utilization of Subtropical Agrobioresources/Guangdong Provincial Key Laboratory of Postharvest Science of Fruits and Vegetables/Guangdong Vegetables Engineering Research Center, College of Horticulture, South China Agricultural University, 510642

Guangzhou, China. ${ }^{2}$ The State Agriculture Ministry Laboratory of Horticultural Plant Growth, Development and Quality Improvement, College of Agriculture and Biotechnology, Zhejiang University, Zijingang Campus, 310058 Hangzhou, China

\section{Conflict of interest}

The authors declare that they have no conflict of interest.

Supplementary Information accompanies this paper at https://doi.org/ 10.1038/s41438-018-0028-z.

Received: 22 November 2017 Revised: 23 February 2018 Accepted: 25 February 2018

Published online: 01 May 2018

\section{References}

1. Hörtensteiner, S. \& Feller, U. Nitrogen metabolism and remobilization during senescence. J. Exp. Bot. 53, 927-937 (2002).

2. Lim, P. O., Kim, H. J. \& Nam, H. G. Leaf senescence. Ann. Rev. Plant Biol. 58, 115-136 (2007).

3. Kim, J., Woo, H. R. \& Nam, H. G. Toward systems understanding of leaf senescence: an integrated multi-omics perspective on leaf senescence research. Mol. Plant 9, 813-825 (2016). 
4. Wu, X. Y., Kuai, B. K., Jia, J. Z. \& Jing, H. C. Regulation of leaf senescence and crop genetic improvement. J. Integr. Plant Biol. 54, 936-952 (2012).

5. Guo, Y. \& Gan, S. S. Translational researches on leaf senescence for enhancing plant productivity and quality. J. Exp. Bot. 65, 3901-3913 (2014).

6. Al Ubeed, H. M. S., Wills, R. B. H., Bowyer, M. C., Vuong, Q. V. \& Golding, J. B. Interaction of exogenous hydrogen sulphide and ethylene on senescence of green leafy vegetables. Postharvest Biol. Technol. 133, 81-87 (2017).

7. Fan, Z. Q. et al. BrWRKY65, a WRKY transcription factor, is involved in regulating three leaf senescence-associated genes in Chinese flowering cabbage. Int. J. Mol. Sci. 18, pii: E1228 (2017).

8. Jibran, R., A Hunter, D. \& P. Dijkwel, P. Hormonal regulation of leaf senescence through integration of developmental and stress signals. Plant Mol. Biol. 82, 547-561 (2013).

9. Schippers, J. H., Schmidt, R., Wagstaff, C. \& Jing, H. C. Living to die and dying to live: the survival strategy behind leaf senescence. Plant Physiol. 169, 914-930 (2015).

10. Hu, Y. et al. Jasmonate regulates leaf senescence and tolerance to cold stress: crosstalk with other phytohormones. J. Exp. Bot. 68, 1361-1369 (2017).

11. He, Y., Fukushige, H., Hildebrand, D. F. \& Gan, S. Evidence supporting a role of jasmonic acid in Arabidopsis leaf senescence. Plant Physiol. 128, 876-884 (2002).

12. Shan, $X$. et al. The role of Arabidopsis Rubisco activase in jasmonate-induced leaf senescence. Plant Physiol. 155, 751-764 (2011).

13. Jiang, Y., Liang, G., Yang, S. \& Yu, D. Arabidopsis WRKY57 functions as a node of convergence for jasmonic acid- and auxin-mediated signaling in jasmonic acid-induced leaf senescence. Plant Cell 26, 230-245 (2014).

14. Qi, T. et al. Regulation of jasmonate-induced leaf senescence by antagonism between bHLH subgroup IIle and IIId factors in Arabidopsis. Plant Cell 27, 1634-1649 (2015).

15. Ueda, J. \& Kato, J. Isolation and identification of a senescence-promoting substance from wormwood (Artemisia absinthium L.). Plant Physiol. 66, 246-249 (1980)

16. Parthier, B. Jasmonates: hormonal regulators or stress factors in leaf senescence. J. Plant Growth Regul. 9, 445-454 (1990).

17. Yan, Y. et al. Disruption of OPR7 and OPR8 reveals the versatile functions of jasmonic acid in maize development and defense. Plant Cell 24, 1420-1436 (2012).

18. Lee, S. H. et al. Mutation of Oryza sativa coronatine insensitive $1 \mathrm{~b}$ (OsCOl1b) delays leaf senescence. J. Integr. Plant Biol. 57, 562-576 (2015).

19. Zhu, X. et al. Jasmonic acid promotes degreening via MYC2/3/4- and ANAC019/055/072-mediated regulation of major chlorophyll catabolic genes. Plant J. 84, 597-610 (2015).

20. Wasternack, C. \& Hause, B. Jasmonates: biosynthesis, perception, signal transduction and action in plant stress response, growth and development. An update to the 2007 review in Annals of Botany. Ann. Bot. 111, 1021-1058 (2013).

21. Goossens, J., Fernández-Calvo, P., Schweizer, F. \& Goossens, A. Jasmonates: signal transduction components and their roles in environmental stress responses. Plant Mol. Biol. 91, 673-689 (2016).

22. Wasternack, C. \& Song, S. Jasmonates: biosynthesis, metabolism, and signaling by proteins activating and repressing transcription. J. Exp. Bot. 68, 1303-1321 (2017).

23. Sasaki-Sekimoto, $Y$. et al. Basic helix-loop-helix transcription factors JASMONATE-ASSOCIATED MYC2-LIKE1 (JAM1), JAM2, and JAM3 are negative regulators of jasmonate responses in Arabidopsis. Plant Physiol. 163, 291-304 (2013).

24. Song, $\mathrm{S}$. et al. ThebHLH subgroup IIId factors negatively regulate jasmonatemediated plant defense and development. PLoS Genet. 9, e1003653 (2013).

25. Song, S. et al. The Jasmonate-ZIM domain proteins interact with the R2R3MYB transcription factors MYB21 and MYB24 to affect Jasmonate-regulated stamen development in Arabidopsis. Plant Cell 23, 1000-1013 (2011).

26. Qi, T. et al. Arabidopsis DELLA and JAZ proteins bind the WD-repeat/bHLH/ MYB complex to modulate gibberellin and jasmonate signaling synergy. Plant Cell 26, 1118-1133 (2014).

27. Schommer, $\mathrm{C}$. et al. Control of jasmonate biosynthesis and senescence by miR319 targets. PLoS Biol. 6, e230 (2008).

28. Danisman, S. et al. Arabidopsis class I and class II TCP transcription factors regulate jasmonic acid metabolism and leaf development antagonistically. Plant Physiol. 159, 1511-1523 (2012).

29. Koyama, T. The roles of ethylene and transcription factors in the regulation of onset of leaf senescence. Front. Plant Sci. 5, 650 (2014).
30. Schippers, J. H. Transcriptional networks in leaf senescence. Curr. Opin. Plant Biol. 27, 77-83 (2015).

31. Miao, Y. \& Zentgraf, U. The antagonist function of Arabidopsis WRKY53 and ESR/ESP in leaf senescence is modulated by the jasmonic and salicylic acid equilibrium. Plant Cell 19, 819-830 (2007).

32. Besseau, S., Li, J. \& Palva, E. T. WRKY54 and WRKY70 cooperate as negative regulators of leaf senescence in Arabidopsis thaliana. J. Exp. Bot. 63, 2667-2679 (2012).

33. Müller, M. \& Munné-Bosch, S. Ethylene response factors: a key regulatory hub in hormone and stress signaling. Plant Physiol. 169, 32-41 (2015).

34. Zhou, M. \& Memelink, J. Jasmonate-responsive transcription factors regulating plant secondary metabolism. Biotechnol. Adv. 34, 441-449 (2016).

35. Ombra, M. N. et al. Biochemical and biological characterization of two Brassicaceae after their commercial expiry date. Food Chem. 218, 335-340 (2017).

36. Zhang, $X$. et al. Correlation of leaf senescence and gene expression/activities of chlorophyll degradation enzymes in harvested Chinese flowering cabbage (Brassica rapa var. parachinensis). J. Plant Physiol. 168, 2081-2087 (2011).

37. Behr, M., Humbeck, K., Hause, G., Deising, H. B. \& Wirsel, S. G. The hemibiotroph Colletotrichum graminicola locally induces photosynthetically active green islands but globally accelerates senescence on aging maize leaves. Mol. Plant Microbe Interact. 23, 879-892 (2010).

38. Zhang, C. M. \& Huang, Z. Effects of endogenous abscisic acid, jasmonic acid, polyamines, and polyamine oxidase activity in tomato seedlings under drought stress. Sci. Hortic. 159, 172-177 (2013).

39. Sainsbury, F., Thuenemann, E. C. \& Lomonossoff, G. P. pEAQ: versatile expression vectors for easy and quick transient expression of heterologous proteins in plants. Plant Biotechnol. J. 7, 682-693 (2009)

40. Cheng, M. N. et al. The WRKY transcription factor HpWRKY44 regulates CytP450-likel expression in red pitaya fruit (Hylocereus polyrhizus). Hortic. Res. 4, 17039 (2017).

41. Hellens, R. P. et al. Transient expression vectors for functional genomics, quantification of promoter activity and RNA silencing in plants. Plant Methods 1, 13 (2005).

42. Yin, $X$. R. et al. Involvement of an ethylene response factor in chlorophyll degradation during citrus fruit degreening. Plant J. 86, 403-412 (2016).

43. Zhang, J. et al. Functional characterization and hormonal regulation of the PHEOPHYTINASE gene LPPPH controlling leaf senescence in perennial ryegrass. J. Exp. Bot. 67, 935-945 (2016).

44. Liu, Q. et al. Two transcription factors, DREB1 and DREB2, with an EREBP/AP2 DNA binding domain separate two cellular signal transduction pathways in drought- and lowtemperature-responsive gene expression, respectively, in Arabidopsis. Plant Cell 10, 391-406 (1998).

45. Tang, M., Sun, J., Liu, Y., Chen, F. \& Shen, S. Isolation and functional characterization of the JCERF gene, a putative AP2/EREBP domain-containing transcription factor, in the woody oil plant Jatropha curcas. Plant Mol. Biol. 63, 419-428 (2007).

46. $\mathrm{Xu}, \mathrm{Z}$. S. et al. Isolation and molecular characterization of the Triticum aestivum L. ethylene-responsive factor 1 (TaERF1) that increases multiple stress tolerance. Plant Mol. Biol. 65, 719-732 (2007).

47. Uji, Y., Akimitsu, K. \& Gomi, K. Identification of OsMYC2-regulated senescenceassociated genes in rice. Planta 245, 1241-1246 (2017).

48. van der Fits, L. \& Memelink, J. ORCA3, a jasmonate-responsive transcriptional regulator of plant primary and secondary metabolism. Science $\mathbf{2 8 9}, \mathbf{2 9 5 - 2 9 7}$ (2000).

49. Yu, Z. X. et al. The jasmonate-responsive AP2/ERF transcription factors AaERF1 and AaERF2 positively regulate artemisinin biosynthesis in Artemisia annua $\mathrm{L}$. Mol. Plant 5, 353-365 (2012).

50. Goossens, J., Mertens, J. \& Goossens, A. Role and functioning of bHLH transcription factors in jasmonate signalling. J. Exp. Bot. 68, 1333-1347 (2017).

51. $\mathrm{Yu}, \mathrm{J}$. et al. JAZ7 negatively regulates dark-induced leaf senescence in Arabidopsis. J. Exp. Bot. 67, 751-762 (2016).

52. Palatnik, J. F. et al. Control of leaf morphogenesis by microRNAs. Nature $\mathbf{4 2 5}$ 257-263 (2003)

53. Li, T. et al. The jasmonate-activated transcription factor MdMYC2 regulates ETHYLENE RESPONSE FACTOR and ethylene biosynthetic genes to promote ethylene biosynthesis during apple fruit ripening. Plant Cell 29, 1316-1334 (2017).

54. Breeze, E. et al. High-resolution temporal profiling of transcripts during Arabidopsis leaf senescence reveals a distinct chronology of processes and regulation. Plant Cell 23, 873-894 (2011). 